\title{
Normal Family of Meromorphic Functions concerning Shared Values
}

\author{
Wei Chen, ${ }^{1}$ Honggen Tian, ${ }^{1}$ Yingying Zhang, ${ }^{1}$ and Wenjun Yuan ${ }^{2}$ \\ ${ }^{1}$ College of Mathematics and System Science, Xinjiang University, Urumqi 830054, China \\ ${ }^{2}$ School of Mathematics and Information Sciences, Guangzhou University, Guangzhou 510006, China
}

Correspondence should be addressed to Wenjun Yuan; wjyuan1957@126.com

Received 27 October 2012; Accepted 13 December 2012

Academic Editor: Bao Qin Li

Copyright (C) 2013 Wei Chen et al. This is an open access article distributed under the Creative Commons Attribution License, which permits unrestricted use, distribution, and reproduction in any medium, provided the original work is properly cited.

We obtain a normal criterion of meromorphic functions concerning, shared values. Let $\mathscr{F}$ be a family of meromorphic functions in a domain $D$ and let $k, n \geq k+2$ be positive integers. Let $a \neq 0, b$ be two finite complex constants. If, for each $f \in \mathscr{F}$, all zeros of $f$ have multiplicity at least $k+1$ and $f+a\left(f^{(k)}\right)^{n}$ and $g+a\left(g^{(k)}\right)^{n}$ share $b$ in $D$ for every pair of functions $f, g \in \mathscr{F}$, then $\mathscr{F}$ is normal in $D$. This result generalizes the related theorem according to Xu et al. and Qi et al., respectively. There is a gap in the proofs of Lemma 3 by Wang (2012) and Theorem 1 by Zhang (2008), respectively. They did not consider the case of $f(z)$ being zerofree. We will fill the gap in this paper.

\section{Introduction and Main Results}

We use $\mathbf{C}$ to denote the open complex plane, $\widehat{\mathbf{C}}(=\mathbf{C} \cup\{\infty\})$ to denote the extended complex plane, and $D$ to denote a domain in C. A family $\mathscr{F}$ of meromorphic functions defined in $D \subset \mathbf{C}$ is said to be normal, if for any sequence $\left\{f_{n}\right\} \subset$ $\mathscr{F}$ contains a subsequence which converges spherically, and locally, uniformly in $D$ to a meromorphic function or $\infty$. Clearly, $\mathscr{F}$ is said to be normal in $D$ if and only if it is normal at every point of $D$ (see $[1,2])$.

Let $h$ be a meromorphic function in a domain $D \subseteq \mathbf{C}$. We say that $h$ is a normal function if there exists a positive number $M$ such that $h^{\sharp}(z) \geq M$ for all $z \in D$, where $h^{\sharp}(z)=$ $\left|h^{\prime}(z)\right| /\left(1+\left.h(z)\right|^{2}\right)$ denotes the spherical derivative of $h$.

Let $D$ be a domain in $\mathbf{C}$ and let $f$ and $g$ be two nonconstant meromorphic functions in $D$. Let $a$ and $b$ be two complex numbers. If $g(z)=b$ whenever $f(z)=a$, we write that

$$
f(z)=a \Longrightarrow g(z)=b .
$$

If $f(z)=a \Rightarrow g(z)=b$ and $(z)=b \Rightarrow f(z)=a$, we write that

$$
f(z)=a \Longleftrightarrow g(z)=b .
$$

If $f(z)=a \Leftrightarrow g(z)=a$, we say that $f$ and $g$ share $a$ (ignoring multiplicities) on $D$. When $a=\infty, f=a$ means $1 / f=0$ (see [2]).

Influenced from Bloch's principle [3], every condition which reduces a meromorphic function in the plane $\mathbf{C}$ to a constant makes a family of meromorphic functions in a domain $D$ normal. Although the principle is false in general (see [4]), many authors proved normality criterion for families of meromorphic functions corresponding to Liouville-Picard type theorem (see $[2,5,6])$.

It is also more interesting to find normality criteria from the point of view of shared values. In this area, Schwick [7] first proved an interesting result that a family of meromorphic functions in a domina is normal if in which every function shares three distinct finite complex numbers with its first derivative. And later, Sun [8] proved that a family of meromorphic functions in a domina is normal if in which each pair of functions share three fixed distinct values, which is an improvement of the famous Montel's Normal Criterion [9] by the ideas of shared values. More results about normality criteria concerning shared values can be found, for instance, see $[10-13]$ and so on.

In 1959, Hayman [14] proved that let $f$ be a meromorphic function in $\mathbf{C}$, if $f^{\prime}-a f^{n} \neq b$, where $n$ is a positive integer and 
$a, b$ are two finite complex numbers such that $n \geq 5$ and $a \neq 0$, then $f$ is a constant. On the other hand, Mues [15] showed that for $n=3,4$, the conclusion is not valid.

The following theorem confirmed a Hayman's wellknown conjecture about normal families in [16].

Theorem A. Let $\mathscr{F}$ be a family of meromorphic functions in $D$, $n$ a positive integer, and $a, b$ two finite complex numbers such that $a \neq 0$. If $n \geq 3$ and for each function $f \in \mathscr{F}, f^{\prime}-a f^{n} \neq b$, then $\mathscr{F}$ is normal in $D$.

In 2008, by the ideas of shared values, Zhang [13] proved the following result.

Theorem B. Let $\mathscr{F}$ be a family of meromorphic functions in $D$, $n$ a positive integer, and $a, b$ two finite complex numbers such that $a \neq 0$. If $n \geq 4$ and for every pair of functions $f$ and $g$ in $\mathscr{F}$, if $f^{\prime}-a f^{n}$ and $g^{\prime}-a g^{n}$ share the value $b$, then $\mathscr{F}$ is normal in $D$.

In 1994, Ye [17] considered a similar problem and obtained that if $f$ is a transcendental meromorphic function and $a$ is a nonzero finite complex number, then $f+a\left(f^{\prime}\right)^{n}$ assumes every finite complex value infinitely often for $n \geq 3$. Ye [17] also asked whether the conclusion remains valid for $n=2$.

In 2008, Fang and Zalcman [18] solved this problem and obtained the following result.

Theorem C. Let $f$ be a transcendental meromorphic function and let $a$ be a nonzero complex number. Then, $f+a\left(f^{\prime}\right)^{n}$ assumes every complex value infinitely often for each positive integer $n \geq 2$.

Remark 1. By a special example, Fang and Zalcman [18] showed Theorem $\mathrm{C}$ is not valid for $n=1$.

On the basis of the previous results, Fang and Zalcman [18] obtained the normality criterion corresponding to Theorem C.

Theorem D. Let $\mathscr{F}$ be a family of meromorphic functions in $D$. Let $n(n \geq 2)$ be an integer and let $a(a \neq 0), b$ be two finite complex numbers. If, for each $f \in \mathscr{F}$, all zeros of $f$ are multiple and $f+a\left(f^{\prime}\right)^{n} \neq b$ on $D$, then $\mathscr{F}$ is normal in $D$.

With the ideas of shared values, Qi and Zhu [19] and Wang [20] extended Theorem D and obtained the following result.

Theorem E. Let $\mathscr{F}$ be a family of meromorphic functions in $D$, all of whose zeros are multiple. Let $n(n \geq 2)$ be an integer and $a, b$ two nonzero finite complex numbers. If $f+a\left(f^{\prime}\right)^{n}$ and $g+a\left(g^{\prime}\right)^{n}$ share $b$ on $D$ for every pair of functions $f, g \in \mathscr{F}$, then $\mathscr{F}$ is normal in $D$.

It is natural to ask whether the condition $f^{\prime}$ can be replaced by $f^{(k)}$ in the previous theorems.

In 2009, $\mathrm{Xu}$ et al. [21] considered the case that $f^{\prime}$ is replaced by the $k$ th derivative $f^{(k)}$ and proved the following results.
Theorem F. Let $f$ be a transcendental meromorphic function on $\mathbf{C}$, a be a nonzero finite complex number, and $n$ and $k$ two positive integers. If $n \geq k+1$, then $f+a\left(f^{(k)}\right)^{n}$ assumes each value $b \in \mathbf{C}$ infinitely often.

Theorem G. Let $a(\neq 0), b \in \mathbf{C}$ and $n$ and $k$ be two positive integers such that $n \geq k+1$. Let $\mathscr{F}$ be a family of meromorphic functions defined on a domain $D$. If $f$ has only zeros of multiplicity at least $k+1$ and $f+a\left(f^{(k)}\right)^{n} \neq b$ in $D$ for every function $f \in \mathscr{F}$, then $\mathscr{F}$ is normal.

In this paper, we study the previous problem and get the following results.

Theorem 2. Let $\mathscr{F}$ be a family of meromorphic functions in the plane domain $D$ and $k, n \geq k+2$ positive integers. Let $a \neq 0, b$ be two finite complex constants. If all zeros of $f$ have multiplicity at least $k+1$ for each $f \in \mathscr{F}$ and $f+a\left(f^{(k)}\right)^{n}$ and $g+a\left(g^{(k)}\right)^{n}$ share $b$ in $D$ for every pair of functions $f, g \in \mathscr{F}$, then $\mathscr{F}$ is normal in $D$.

Theorem 3. Let $\mathscr{F}$ be a family of meromorphic functions in the plane domain $D$ and $k, n \geq k+2$ positive integers. Let $a \neq 0, b, c$ be finite complex constants. If all zeros of $f$ have multiplicity at least $k$ and $f(z)=0 \Rightarrow(f(z))^{(k)}=c$ for each $f \in \mathscr{F} f+$ $a\left(f^{(k)}\right)^{n}$ and $g+a\left(g^{(k)}\right)^{n}$ share $b$ in $D$ for every pair of functions $f, g \in \mathscr{F}$, then $\mathscr{F}$ is normal in $D$.

Example 4. Let $D=\{z:|z|<1\}$ and $\mathscr{F}=\left\{f_{n}(z)\right\}$, where

$$
f_{n}(z)=\frac{n}{(k+1) !} z^{k+1}, \quad z \in D, n=1,2, \ldots,
$$

then

$$
f_{n}(z)+a\left(f_{n}^{(k)}(z)\right)^{k+1}=\left(\frac{n}{(k+1) !}+a n^{k+1}\right) z^{k+1} .
$$

Clearly, for each pair of functions, $f, g, f+a\left(f^{(k)}\right)^{k+1}$ and $g+a\left(g^{(k)}\right)^{k+1}$ share the value 0 in $D$; however, $\mathscr{F}$ is not normal in $D$.

Example 5. Let $k=1, a=1, D=\{z:|z|<1\}$, and $\mathscr{F}=$ $\left\{f_{n}(z)\right\}$, where

$$
f_{n}(z)=n z-n^{3}, \quad z \in D, n=1,2, \ldots,
$$

then

$$
f_{n}(z)+a\left(f_{n}^{\prime}(z)\right)^{3}=n z
$$

Clearly, for each pair of functions, $f, g, f+a\left(f^{\prime}\right)^{n}$ and $g+$ $a\left(g^{\prime}\right)^{n}$ share the value 0 in $D$; however, $\mathscr{F}$ is not normal in $D$.

Remark 6. Example 4 shows that the condition $n \geq k+2$ in Theorem 2 is sharp. And Example 5 shows that the condition in which all zeros of $f$ have multiplicity at least $k+1$ in Theorem 2 is sharp.

Remark 7. There is a gap in the proofs of Theorems 1.2 and 1.5 by Zhang [13] and Wang [20], respectively. They did not 
consider the case of $f(z)$ being zero free in the proof of both Lemma 3 in [20] and Theorem 1 in [13], respectively. In Section 2, both Lemmas 9 and 11 fill the gap in their proofs of both Lemma 3 in [20] and Theorem 1 in [13], respectively.

\section{Preliminary Lemmas}

In order to prove our theorems, we need the following lemmas.

First, we need the following well-known Pang-Zalcman lemma, which is the local version of $[11,22]$.

Lemma 8. Let $\mathscr{F}$ be a family of meromorphic functions in a domain $D$ and $k$ a positive integer, such that each function $f \in$ $\mathscr{F}$ has only zeros of multiplicity at least $k$, and suppose that there exists $A \geq 1$ such that $\left|f^{(k)}(z)\right| \leq A$ whenever $f(z)=$ $0, f \in \mathscr{F}$. If $\mathscr{F}$ is not normal at $z_{0} \in D$, then for each $0 \leq \alpha \leq k$, there exist a sequence of points $z_{n} \in D, z_{n} \rightarrow z_{0}$, a sequence of positive numbers $\rho_{n} \rightarrow 0^{+}$, and a subsequence of functions $f_{n} \in \mathscr{F}$ such that

$$
g_{n}(\zeta)=\frac{f_{n}\left(z_{n}+\rho_{n} \varsigma\right)}{\rho_{n}^{\alpha}} \longrightarrow g(\zeta),
$$

locally uniformly with respect to the spherical metric in $\mathrm{C}$. Here, $g$ is a nonconstant meromorphic function such that $g^{\sharp}(\zeta) \leq$ $g^{\sharp}(0)=k A+1$ and all of whose zeros have multiplicity at least $k$. Moreover, $g$ has an order at most 2. If $g$ is a holomorphic function, then $g$ is of exponential type and has an order at most 1.

Lemma 9 (see [23]). Let $f$ be a nonconstant zero-free rational function, $n \geq 2, k$ two positive integers, and $a \neq 0, b$ two complex constants. Then, the function $f+a\left(f^{(k)}\right)^{n}-b$ has at least $n k+1$ distinct zeros in $\mathbf{C}$.

Lemma 10. Let $k, n \geq k+2$ be two positive integers and let $a \neq 0$ be a constant. Let $f$ be a nonconstant rational meromorphic function, all zeros of $f$ have multiplicity at least $k+1$, then $f+a\left(f^{(k)}\right)^{n}$ has at least two distinct zeros.

Proof. When $f$ is a nonconstant polynomial, noting that $n \geq$ $k+2$ and all zeros of $f$ have multiplicity at least $k+1$, we know that $f+a\left(f^{(k)}\right)^{n}$ must have zero. Hence, $f+a\left(f^{(k)}\right)^{n}$ has exactly one zero $z_{0}$. Set that

$$
f+a\left(f^{(k)}\right)^{n}=A\left(z-z_{0}\right)^{l}
$$

where $A$ is a nonzero constant and $l$ is a positive integer such that $l \geq k+1$.

It follows from (8) that

$$
\begin{aligned}
f^{(k)}+a\left(\left(f^{(k)}\right)^{n}\right)^{(k)} & =f^{(k)}(1+a F(z)) \\
& =A l(l-1) \cdots(l-k+1)\left(z-z_{0}\right)^{l-k}
\end{aligned}
$$

where $F(z)=n(n-1) \cdots(n-k+1)\left(f^{(k)}\right)^{n-k-1}\left(f^{(k+1)}\right)^{k}+\cdots+$ $n\left(f^{(k)}\right)^{n-1} f^{(2 k)}$. Since all zeros of $f$ have multiplicity at least $k+1$ and $f^{(k)}$ has only one zero $z_{0}$, we can deduce from (9) that $\mathrm{f}$ has only zero $z_{0}$. Writing that $f(z)=B\left(z-z_{0}\right)^{m}$, where $m$ is an integer such that $m \geq k+1$, then

$$
\begin{aligned}
f+a\left(f^{(k)}\right)^{n} & =B\left(z-z_{0}\right)^{m}+a\left[\left(B\left(z-z_{0}\right)^{m}\right)^{(k)}\right]^{n} \\
& =B\left(z-z_{0}\right)^{m}\left[1+a B^{n-1} C^{n}\left(z-z_{0}\right)^{n(m-k)-m}\right],
\end{aligned}
$$

where $C=m(m-1) \cdots(m-k+1)$. Equation (9) combing inequality that $n(m-k)-m \geq(n-1)(k+1)-n k=$ $n-k-1 \geq 1$ gives that $f+a\left(f^{(k)}\right)^{n}$ has at least two distinct zeros, a contradiction.

When $f$ is rational but not a polynomial, we consider two cases.

Case 1. If $f+a\left(f^{(k)}\right)^{n}$ has exactly one zero, then suppose that $f+a\left(f^{(k)}\right)^{n}$ has only zero $z_{0}$ with multiplicity at least $l$. If $f \neq 0$, by Lemma 9, we get a contradiction. So $f$ has zeros, then we can deduce that $z_{0}$ is the only zero of $f$. Otherwise, $f+a\left(f^{(k)}\right)^{n}$ has at least two zeros. Set that

$$
f(z)=\frac{A\left(z-z_{0}\right)^{m}}{\left(z-z_{1}\right)^{\beta_{1}}\left(z-z_{2}\right)^{\beta_{2}} \cdots\left(z-z_{t}\right)^{\beta_{t}}},
$$

where $A$ is a nonzero constant and $m \geq k+1$. For simplicity, we denote that

$$
\beta_{1}+\beta_{2}+\cdots+\beta_{t}=q
$$

From (11), we have

$$
f^{(k)}=\frac{A\left(z-z_{0}\right)^{m-k} g(z)}{\left(z-z_{1}\right)^{\beta_{1}+k}\left(z-z_{2}\right)^{\beta_{2}+k} \cdots\left(z-z_{t}\right)^{\beta_{t}+k}},
$$

where $g(z)=(m-q)(m-q-1) \cdots(m-q-k+1) z^{k t}+\cdots+C$ is polynomial and $C$ is a constant.

By (11) and (13), we have

$$
\begin{aligned}
f & +a\left(f^{(k)}\right)^{n} \\
= & \frac{A\left(z-z_{0}\right)^{m}}{\left(z-z_{1}\right)^{\beta_{1}}\left(z-z_{2}\right)^{\beta_{2}} \cdots\left(z-z_{t}\right)^{\beta_{t}}} \\
& +\frac{a A^{n}\left(z-z_{0}\right)^{n(m-k)} g^{n}(z)}{\left(z-z_{1}\right)^{n\left(\beta_{1}+k\right)}\left(z-z_{2}\right)^{n\left(\beta_{2}+k\right)} \cdots\left(z-z_{t}\right)^{n\left(\beta_{t}+k\right)}} \\
= & \frac{A\left(z-z_{0}\right)^{m}\left(z-z_{1}\right)^{(n-1) \beta_{1}+n k} \cdots\left(z-z_{t}\right)^{(n-1) \beta_{t}+n k}}{\left(z-z_{1}\right)^{n\left(\beta_{1}+k\right)}\left(z-z_{2}\right)^{n\left(\beta_{2}+k\right)} \cdots\left(z-z_{t}\right)^{n\left(\beta_{t}+k\right)}} \\
& +\frac{a A^{n}\left(z-z_{0}\right)^{n(m-k)} g^{n}(z)}{\left(z-z_{1}\right)^{n\left(\beta_{1}+k\right)}\left(z-z_{2}\right)^{n\left(\beta_{2}+k\right)} \cdots\left(z-z_{t}\right)^{n\left(\beta_{t}+k\right)}} .
\end{aligned}
$$


Since $n \geq k+2, m \geq k+1$, we deduce that $n(m-k)-m \geq$ $(n-1)(k+1)-n k=n-k-1 \geq 1$, and then

$$
\begin{aligned}
f+ & a\left(f^{(k)}\right)^{n} \\
= & A\left(z-z_{0}\right)^{m}\left[\left(z-z_{1}\right)^{(n-1) \beta_{1}+n k} \cdots\left(z-z_{t}\right)^{(n-1) \beta_{t}+n k}\right. \\
& \left.\quad+a A^{n-1}\left(z-z_{0}\right)^{n(m-k)-m} g^{n}(z)\right] \\
& \times\left(\left(z-z_{1}\right)^{n\left(\beta_{1}+k\right)}\left(z-z_{2}\right)^{n\left(\beta_{2}+k\right)} \cdots\left(z-z_{t}\right)^{n\left(\beta_{t}+k\right)}\right)^{-1} .
\end{aligned}
$$

By the assumption that $f+a\left(f^{(k)}\right)^{n}$ has exactly one zero $z_{0}$ with multiply $l$ and (15), we get

$$
\begin{aligned}
f & +a\left(f^{(k)}\right)^{n} \\
& =\frac{C\left(z-z_{0}\right)^{l}}{\left(z-z_{1}\right)^{n\left(\beta_{1}+k\right)}\left(z-z_{2}\right)^{n\left(\beta_{2}+k\right)} \cdots\left(z-z_{t}\right)^{n\left(\beta_{t}+k\right)}},
\end{aligned}
$$

where $C$ is a nonzero constant. Both (15) and (16) imply that

$$
\begin{aligned}
& C\left(z-z_{0}\right)^{l} \\
& \equiv A\left(z-z_{0}\right)^{m}\left[\left(z-z_{1}\right)^{(n-1) \beta_{1}+n k} \cdots\left(z-z_{t}\right)^{(n-1) \beta_{t}+n k}\right. \\
& \left.\quad+a A^{n-1}\left(z-z_{0}\right)^{n(m-k)-m} g^{n}(z)\right] .
\end{aligned}
$$

Case 1.1. If $l>m$, from (17), we can deduce that $z_{0}$ is a zero of $\left(z-z_{1}\right)^{(n-1) \beta_{1}+n k} \cdots\left(z-z_{t}\right)^{(n-1) \beta_{t}+n k}$, a contradiction.

Case 1.2. If $l=m$, from (17), it follows that

$$
\begin{aligned}
& \left(z-z_{1}\right)^{(n-1) \beta_{1}+n k} \cdots\left(z-z_{t}\right)^{(n-1) \beta_{t}+n k} \\
& \quad+a A^{n-1}\left(z-z_{0}\right)^{n(m-k)-m} g^{n}(z)=\frac{C}{A} .
\end{aligned}
$$

For simplicity, we denote that

$$
\begin{gathered}
g_{1}(z)=\left(z-z_{1}\right)^{(n-1) \beta_{1}+n k} \cdots\left(z-z_{t}\right)^{(n-1) \beta_{t}+n k}, \\
g_{2}(z)=a A^{n-1}\left(z-z_{0}\right)^{n(m-k)-m} g^{n}(z) .
\end{gathered}
$$

Case 1.2.1. If $q \geq m-k$, then

$$
\begin{aligned}
\operatorname{deg}\left(g_{1}\right) & =(n-1)\left(\beta_{1}+\beta_{2}+\cdots+\beta_{t}\right)+n k t \\
& =(n-1) q+n k t \\
& \geq(n-1)(m-k)+n k t, \\
\operatorname{deg}\left(g_{2}\right) & =n(m-k)-m+n \operatorname{deg}(g) \\
& \leq n(m-k)-m+n k t .
\end{aligned}
$$

Thus, $\operatorname{deg}\left(g_{1}\right)-\operatorname{deg}\left(g_{2}\right) \geq(n-1)(m-k)-n(m-k)+$ $m=k$. Therefore, $g_{1}+g_{2}$ is a non-constant polynomial, a contradiction.

Case 1.2.2. If $q<m-k$, then

$$
\begin{gathered}
\operatorname{deg}\left(g_{1}\right)=(n-1)\left(\beta_{1}+\beta_{2}+\cdots+\beta_{t}\right)+n k t \\
=(n-1) q+n k t \leq(n-1)(m-k-1)+n k t, \\
\operatorname{deg}\left(g_{2}\right)=n(m-k)-m+n \operatorname{deg}(g)=n(m-k)-m+n k t .
\end{gathered}
$$

Hence,

$$
\begin{aligned}
\operatorname{deg}\left(g_{2}\right)-\operatorname{deg}\left(g_{1}\right) & \geq n(m-k)-m-(n-1)(m-k-1) \\
& =n-k-1 \geq 1 .
\end{aligned}
$$

Therefore, $g_{1}+g_{2}$ is a non-constant polynomial, a contradiction.

Case 2. If $f+a\left(f^{(k)}\right)^{n}$ has no zeros, then $l=0$ for (16). It is the same argument as the proof of Case 1 that we have a contradiction.

The proof is complete.

Lemma 11 (see [24]). Let $f$ be a nonconstant zero-free rational function and $k$ a positive integer. Then, $f^{(k)}-1$ has at least $k+1$ distinct zeros.

\section{The Proofs of Theorems}

Proof of Theorem 2. Without loss of generality, we may assume that $D=\Delta=\{z:|z|<1\}$. Suppose, on the contrary, that $\mathscr{F}$ is not normal at $z_{0}$.

Case 1 . When $b=0$, by Lemma 8 , there exist a sequence $\left\{z_{j}\right\}$ of complex numbers with $z_{j} \rightarrow z_{0}$ and a sequence $\left\{\rho_{j}\right\}$ of positive numbers with $\rho_{j} \rightarrow 0$ such that

$$
g_{j}(\xi)=\rho_{j}^{(-n k) /(n-1)} f_{j}\left(z_{j}+\rho_{j} \xi\right) \longrightarrow g(\xi),
$$

locally uniformly on compact subsets of $\mathbf{C}$, where $g(\xi)$ is a non-constant meromorphic function in $\mathbf{C}$, all of whose zeros have multiplicity at least $k+1$. Moreover, $g(\xi)$ has an order at most 2 .

If $g+a\left(g^{(k)}\right)^{n} \equiv 0$, then $g$ has no poles, so $g$ is an entire function. Since all zeros of $g$ have multiplicity at least $k+1$, if $z_{0}$ is a zero of $g$ with multiply $q(\geq k+1)$, then $z_{0}$ is a zero of $\left(g^{(k)}\right)^{n}$ with multiplicity $n(q-k)$, by $g+a\left(g^{(k)}\right)^{n} \equiv 0$. It follows that $g=-a\left(g^{(k)}\right)^{n}$, so $q=(q-k) n$ which implies that $n k \geq(n-1)(k+1)$, then $n \leq k+1$, which contradicts with $n \geq k+2$. So $g$ has no zeros, then we can deduce that $g=e^{c \xi+d}$, where $c \neq 0, d$ are constants. Then,

$$
\begin{aligned}
g+a\left(g^{(k)}\right)^{n} & =e^{c \xi+d}+a\left(c^{k} e^{c \xi+d}\right)^{n} \\
& =e^{c \xi+d}\left(1+a c^{n k} e^{(n-1)(c \xi+d)}\right) \equiv 0 .
\end{aligned}
$$


Hence,

$$
e^{(n-1)(c \xi+d)} \equiv-\frac{1}{a c^{n k}}
$$

which is impossible because $n \geq k+2 \geq 2$.

Theorems F, G, and Lemma 10 imply that $g+a\left(g^{(k)}\right)^{n}$ has at least two distinct zeros; we may assume that there exist two zeros $\xi_{0}, \xi_{0}^{*}$. We choose a positive number $\delta$ small enough such that $D_{1} \cap D_{2}=\emptyset$ and $f+a\left(f^{(k)}\right)^{n}$ has no other zeros in $D_{1} \cup D_{2}$ except for $\xi_{0}$ and $\xi_{0}^{*}$, where

$$
\begin{aligned}
& D_{1}=\left\{\xi \in C|| \xi-\xi_{0} \mid<\delta\right\}, \\
& D_{2}=\left\{\xi \in C|| \xi-\xi_{0}^{*} \mid<\delta\right\} .
\end{aligned}
$$

Since

$$
\begin{aligned}
g_{j}+a\left(g_{j}^{(k)}\right)^{n}= & \rho_{j}^{(-n k) /(n-1)} \\
& \times\left[f_{j}\left(z_{j}+\rho_{j} \xi_{j}\right)\right. \\
& \left.+a\left(f_{j}^{(k)}\left(z_{j}+\rho_{j} \xi_{j}\right)\right)^{n}\right] \longrightarrow g+a\left(g^{(k)}\right)^{n},
\end{aligned}
$$

by Hurwitz's theorem, we know that for sufficiently large $j$ there exist points $\xi_{j} \in D_{1}, \xi_{j}^{*} \in D_{2}$ such that

$$
\begin{aligned}
& f_{j}\left(z_{j}+\rho_{j} \xi_{j}^{*}\right)+a\left[f_{j}^{(k)}\left(z_{j}+\rho_{j} \xi_{j}^{*}\right)\right]^{n}=0, \\
& f_{j}\left(z_{j}+\rho_{j} \xi_{j}\right)+a\left[f_{j}^{(k)}\left(z_{j}+\rho_{j} \xi_{j}\right)\right]^{n}=0 .
\end{aligned}
$$

Note that $f_{m} f_{m}^{(k)}$ and $f_{j} f_{j}^{(k)}$ share $b$, it follows that

$$
\begin{gathered}
f_{m}\left(z_{j}+\rho_{j} \xi_{j}^{*}\right)+a\left[f_{m}^{(k)}\left(z_{j}+\rho_{j} \xi_{j}^{*}\right)\right]^{n}=0, \\
f_{m}\left(z_{j}+\rho_{j} \xi_{j}\right)+a\left[f_{m}^{(k)}\left(z_{j}+\rho_{j} \xi_{j}\right)\right]^{n}=0 .
\end{gathered}
$$

Fix $m$, let $j \rightarrow \infty$, and note that $z_{j}+\rho_{j} \xi_{j} \rightarrow z_{0}, z_{j}+\rho_{j} \xi_{j}^{*} \rightarrow$ $z_{0}$, then we obtain

$$
f_{j}\left(z_{0}\right)+a\left[f_{j}^{(k)}\left(z_{0}\right)\right]^{n}=0 .
$$

Since the zeros of $f_{j}(\xi)+a\left[f_{j}^{(k)}(\xi)\right]^{n}$ have no accumulation point, for sufficiently large $j$, we have

$$
z_{j}+\rho_{j} \xi_{j}=z_{0}, \quad z_{j}+\rho_{j} \xi_{j}^{*}=z_{0}
$$

Hence,

$$
\xi_{j}=\frac{z_{0}-z_{j}}{\rho_{j}}, \quad \xi_{j}^{*}=\frac{z_{0}-z_{j}}{\rho_{j}} .
$$
$\emptyset$.

This contradicts with the facts $\xi_{j} \in D_{1}, \xi_{j}^{*} \in D_{2}, D_{1} \cap D_{2}=$

Case 2. When $b \neq 0$, by Lemma 8 , there exist a sequence $\left\{z_{j}\right\}$ of complex numbers with $z_{j} \rightarrow z_{0}$ and a sequence $\left\{\rho_{j}\right\}$ of positive numbers with $\rho_{j} \rightarrow 0$ such that

$$
g_{j}(\xi)=\rho_{j}^{-k} f_{j}\left(z_{j}+\rho_{j} \xi\right) \longrightarrow g(\xi),
$$

locally uniformly on compact subsets of C, where $g(\xi)$ is a non-constant meromorphic function in $\mathbf{C}$, all of whose zeros have multiplicity at least $k+1$. Moreover, $g(\xi)$ has an order at most 2 .

Case 2.1. If $a\left(g^{(k)}\right)^{n} \equiv b$, then $g$ is a polynomial with a degree at most $k$, this contradicts with the fact that $g$ has only zeros with multiplicity at least $k+1$.

Case 2.2. If $a\left(g^{(k)}\right)^{n} \neq b$, let $c_{1}, c_{2}, \ldots, c_{n}$ be the (distinct) solutions of $w_{n}=b / a$, then $g^{(k)} \neq c_{i}(i=1,2, \ldots, n)$, and then $g^{(k)}$ is a constant by Picard theorem. It follows that $g$ is a polynomial with a degree at most $k$, this contradicts with the fact that $g$ has only zeros with multiplicity at least $k+1$. Therefore, $a\left(g^{(k)}\right)^{n}=b$ has solutions.

We claim that $a\left(g^{(k)}\right)^{n}-b$ has just one zero. Otherwise, we may assume that there exist two zeros $\xi_{0}, \xi_{0}^{*}$. We choose a positive number $\delta$ small enough such that $D_{1} \cap D_{2}=\emptyset$ and such that $f+a\left(f^{(k)}\right)^{n}$ has no other zeros in $D_{1} \cup D_{2}$ except for $\xi_{0}$ and $\xi_{0}^{*}$, where

$$
\begin{aligned}
& D_{1}=\left\{\xi \in C|| \xi-\xi_{0} \mid<\delta\right\}, \\
& D_{2}=\left\{\xi \in C|| \xi-\xi_{0}^{*} \mid<\delta\right\} .
\end{aligned}
$$

Combing

$$
\begin{aligned}
\rho_{j}^{k} g_{j}+a\left(g_{j}^{(k)}\right)^{n}-b= & f_{j}\left(z_{j}+\rho_{j} \xi_{j}\right)+a\left(f_{j}^{(k)}\left(z_{j}+\rho_{j} \xi_{j}\right)\right)^{n} \\
& -b \longrightarrow a\left(g^{(k)}\right)^{n}-b
\end{aligned}
$$

and Hurwitz's theorem, we have that for sufficiently large $j$ there exist points $\xi_{j} \in D_{1}, \xi_{j}^{*} \in D_{2}$ such that

$$
\begin{gathered}
f_{j}\left(z_{j}+\rho_{j} \xi_{j}^{*}\right)+a\left[f_{j}^{(k)}\left(z_{j}+\rho_{j} \xi_{j}^{*}\right)\right]^{n}-b=0, \\
f_{j}\left(z_{j}+\rho_{j} \xi_{j}\right)+a\left[f_{j}^{(k)}\left(z_{j}+\rho_{j} \xi_{j}\right)\right]^{n}-b=0 .
\end{gathered}
$$

From the hypothesis that $f_{m} f_{m}^{(k)}$ and $f_{j} f_{j}^{(k)}$ share $b$, it follows that

$$
\begin{gathered}
f_{m}\left(z_{j}+\rho_{j} \xi_{j}^{*}\right)+a\left[f_{m}^{(k)}\left(z_{j}+\rho_{j} \xi_{j}^{*}\right)\right]^{n}-b=0, \\
f_{m}\left(z_{j}+\rho_{j} \xi_{j}\right)+a\left[f_{m}^{(k)}\left(z_{j}+\rho_{j} \xi_{j}\right)\right]^{n}-b=0 .
\end{gathered}
$$

By letting $m \rightarrow \infty$ and noting that $z_{j}+\rho_{j} \xi_{j} \rightarrow z_{0}, z_{j}+$ $\rho_{j} \xi_{j}^{*} \rightarrow z_{0}$, we obtain

$$
f_{j}\left(z_{0}\right)+a\left[f_{j}^{(k)}\left(z_{0}\right)\right]^{n}-b=0 .
$$

Since the zeros of $f_{j}(\xi)+a\left[f_{j}^{(k)}(\xi)\right]^{n}$ have no accumulation point, for sufficiently large $j$, we have

$$
z_{j}+\rho_{j} \xi_{j}=z_{0}, \quad z_{j}+\rho_{j} \xi_{j}^{*}=z_{0} .
$$

Hence,

$$
\xi_{j}=\frac{z_{0}-z_{j}}{\rho_{j}}, \quad \xi_{j}^{*}=\frac{z_{0}-z_{j}}{\rho_{j}}
$$


which contradicts with the facts $\xi_{j} \in D_{1}, \xi_{j}^{*} \in D_{2}, D_{1} \cap D_{2}=$ $\emptyset$.

Therefore, $a\left(g^{(k)}\right)^{n}-b$ has just one zero. Thus, $g^{(k)}-c_{i}$ has at most one zero for the only one of $i \in\{1,2, \ldots, n\}$. We may assume that $g^{(k)}-c_{1}$ has at most one zero, then $g^{(k)} \neq c_{i}(i=2,3, \ldots, n)$. From the second fundamental theorem of Nevanlinna,

$$
\begin{aligned}
T\left(r, g^{(k)}\right) \leq & \bar{N}\left(r, g^{(k)}\right)+\bar{N}\left(r, \frac{1}{g^{(k)}-c_{2}}\right)+\bar{N}\left(r, \frac{1}{g^{(k)}-c_{3}}\right) \\
& +\cdots+\bar{N}\left(r, \frac{1}{g^{(k)}-c_{n}}\right)+S\left(r, g^{(k)}\right) \\
\leq & \bar{N}\left(r, g^{(k)}\right)+S\left(r, g^{(k)}\right) \\
\leq & \frac{1}{2} N\left(r, g^{(k)}\right)+S\left(r, g^{(k)}\right) \\
\leq & \frac{1}{2} T\left(r, g^{(k)}\right)+S\left(r, g^{(k)}\right) .
\end{aligned}
$$

It follows that $T\left(r, g^{(k)}\right)=S\left(r, g^{(k)}\right)$, which is impossible.

The proof of Theorem 2 is proved.

Proof of Theorem 3. Suppose, to the contrary, that $\mathscr{F}$ is not normal in $\Delta$. Then, there exists at least one point $z_{0}$ such that $\mathscr{F}$ is not normal at the point $z_{0}$. Without loss of generality, we assume that $z_{0}=0$. Then, by Lemma 8 , we can find that $f_{j} \in \mathscr{F}, z_{j} \rightarrow 0$, and $\rho_{j} \rightarrow 0$, such that

$$
g_{j}(\xi)=\rho_{j}^{(-n k) /(n-1)} f_{j}\left(z_{j}+\rho_{j} \xi\right) \longrightarrow g(\xi)
$$

converges locally uniformly with respect to the spherical metric to a nonconstant meromorphic function $g(\zeta)$, and all zeros of $g(\zeta)$ are of multiplicity at least $k$, and the spherical derivative of $g(\zeta)$ is bounded.

Then, we have

$$
g_{j}^{(k)}(\xi)=\rho_{j}^{(-k) /(n-1)}\left(f_{j}\left(z_{j}+\rho_{j} \xi\right)\right)^{(k)} \longrightarrow g^{(k)}(\xi),
$$

also locally uniformly with respect to the spherical metric.

We claim that all zeros of $g(\xi)$ are of multiplicity at least $k+1$. Suppose that $\xi_{0}$ is a zero of $g(\xi)$, then obviously, $g(\xi) \not \equiv$ 0 . From Hurwitz's theorem, there exist $\xi_{j} \rightarrow \xi_{0}$ such that

$$
f_{j}\left(z_{j}+\rho_{j} \xi_{j}\right)=0
$$

then we have $\left(f_{j}\left(z_{j}+\rho_{j} \xi_{j}\right)\right)^{(k)}=c$ by the condition that $f(z)=$ $0 \Rightarrow(f(z))^{(k)}=c$.

If $c=0$, then all zeros of each $f \in \mathscr{F}$ are of multiplicity at least $k+1$ near 0 by the hypothesis. Hence, Theorem 2 tells us that $\mathscr{F}$ is normal at 0 , which contradicts our supposition.

If $c \neq 0$, then

$$
\begin{aligned}
\left(g\left(\xi_{0}\right)\right)^{(k)} & =\lim _{j \rightarrow \infty} g_{j}^{(k)}\left(\xi_{j}\right) \\
& =\lim _{j \rightarrow \infty} \rho_{j}^{(-k) /(n-1)}\left(f_{j}\left(z_{j}+\rho_{j} \xi_{j}\right)\right)^{(k)}=\infty,
\end{aligned}
$$

which is also a contradiction.

The proof of Theorem 3 is completed.

\section{Acknowledgment}

This work was supported by the Visiting Scholar Program of Chern Institute of Mathematics at Nankai University. Zo Yingying would like to express his hearty thanks to Chern Institute of Mathematics which provided him with very comfortable research environments while working as a visiting scholar. This word was also supported by Nature Science Foundation of China (11271090); Nature Science Foundation of Guangdong Province (S2012010010121); Academic technology innovation Foundation of Xinjiang Normal University.

\section{References}

[1] W. K. Hayman, Meromorphic Functions, Clarendon Press, Oxford, UK, 1964.

[2] J. L. Schiff, Normal Families, Springer, Berlin, Germany, 1993.

[3] W. Bergweiler, "Bloch's principle," Computational Methods and Function Theory, vol. 6, no. 1, pp. 77-108, 2006.

[4] L. A. Rubel, "Four counterexamples to Bloch's principle," Proceedings of the American Mathematical Society, vol. 98, no. 2, pp. 257-260, 1986.

[5] D. Drasin, "Normal families and the Nevanlinna theory," Acta Mathematica, vol. 122, no. 1, pp. 231-263, 1969.

[6] L. Yang, Value Distribution Theory, Springer, Berlin, Germany, Science Press, Beijing, China, 1993.

[7] W. Schwick, "Normality criteria for families of meromorphic functions," Journal d'Analyse Mathématique, vol. 52, no. 1, pp. 241-289, 1981.

[8] D. C. Sun, “The shared value criterion for normality," Journal of Wuhan University, no. 3, pp. 9-12, 1994 (Chinese).

[9] P. Montel, "Sur les familles de fonctions analytiques qui admettent des valeurs exceptionnelles dans un domaine (in France)," Annales Scientifiques de l'École Normale Supérieure, vol. 29, pp. 487-535, 1912.

[10] M. Fang and L. Zalcman, "A note on normality and shared values," Journal of the Australian Mathematical Society, vol. 76, no. 1, pp. 141-150, 2004.

[11] X. Pang and L. Zalcman, "Normal families and shared values," Bulletin of the London Mathematical Society, vol. 32, no. 3, pp. 325-331, 2000.

[12] X. Pang and L. Zalcman, "Normality and shared values," Arkiv for Matematik, vol. 38, no. 1, pp. 171-182, 2000.

[13] Q. Zhang, "Normal families of meromorphic functions concerning shared values," Journal of Mathematical Analysis and Applications, vol. 338, no. 1, pp. 545-551, 2008.

[14] W. K. Hayman, "Picard values of meromorphic functions and their derivatives," Annals of Mathematics, vol. 70, pp. 9-42, 1959.

[15] E. Mues, "Über ein Problem von Hayman," Mathematische Zeitschrift, vol. 164, no. 3, pp. 239-259, 1979.

[16] W. K. Hayman, Research Problems in Function Theory, The Athlone Press University of London, London, UK, 1967.

[17] Y. S. Ye, "A Picard type theorem and Bloch law," Chinese Annals of Mathematics B, vol. 15, no. 1, pp. 75-80, 1994 (Chinese).

[18] M. L. Fang and L. Zalcman, "On value distribution of $f+a\left(f^{\prime}\right)^{n}$," Science in China A, vol. 38, pp. 279-285, 2008.

[19] J. Qi and T. Zhu, "Some normal criteria about shared values with their multiplicity zeros," Abstract and Applied Analysis, vol. 2010, Article ID 147878, 14 pages, 2010. 
[20] Y.-M. Wang, "On normality of meromorphic functions with multiple zeros and sharing values," Turkish Journal of Mathematics, vol. 36, no. 2, pp. 263-271, 2012.

[21] Y. Xu, F. Q. Wu, and L. W. Liao, "Picard values and normal families of mero-morphic functions," Proceedings of the Royal Society of Edinburgh, vol. 139A, pp. 1091-1099, 2009.

[22] L. Zalcman, "Normal families: new perspectives," Bulletin of the American Mathematical Society, vol. 35, no. 3, pp. 215-230, 1998.

[23] Y. T. Li, "Normal families of zero-free meromorphic functions," Abstract and Applied Analysis, vol. 2012, Article ID 908123, 12 pages, 2012.

[24] J. M. Chang, "Normality and quasinormality of zero-free meromorphic functions," Acta Mathematica Sinica, vol. 28, no. 4, pp. 707-716, 2012. 


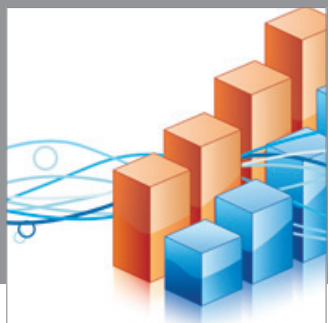

Advances in

Operations Research

mansans

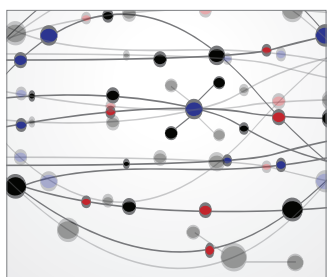

The Scientific World Journal
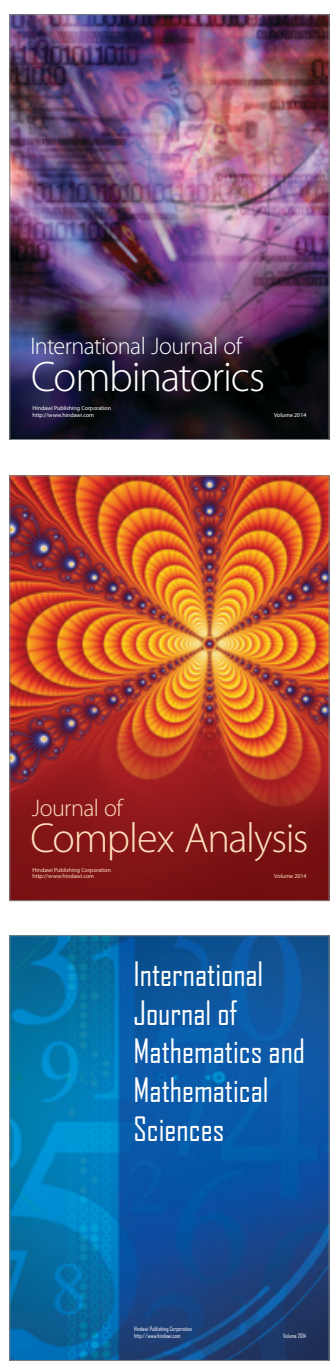
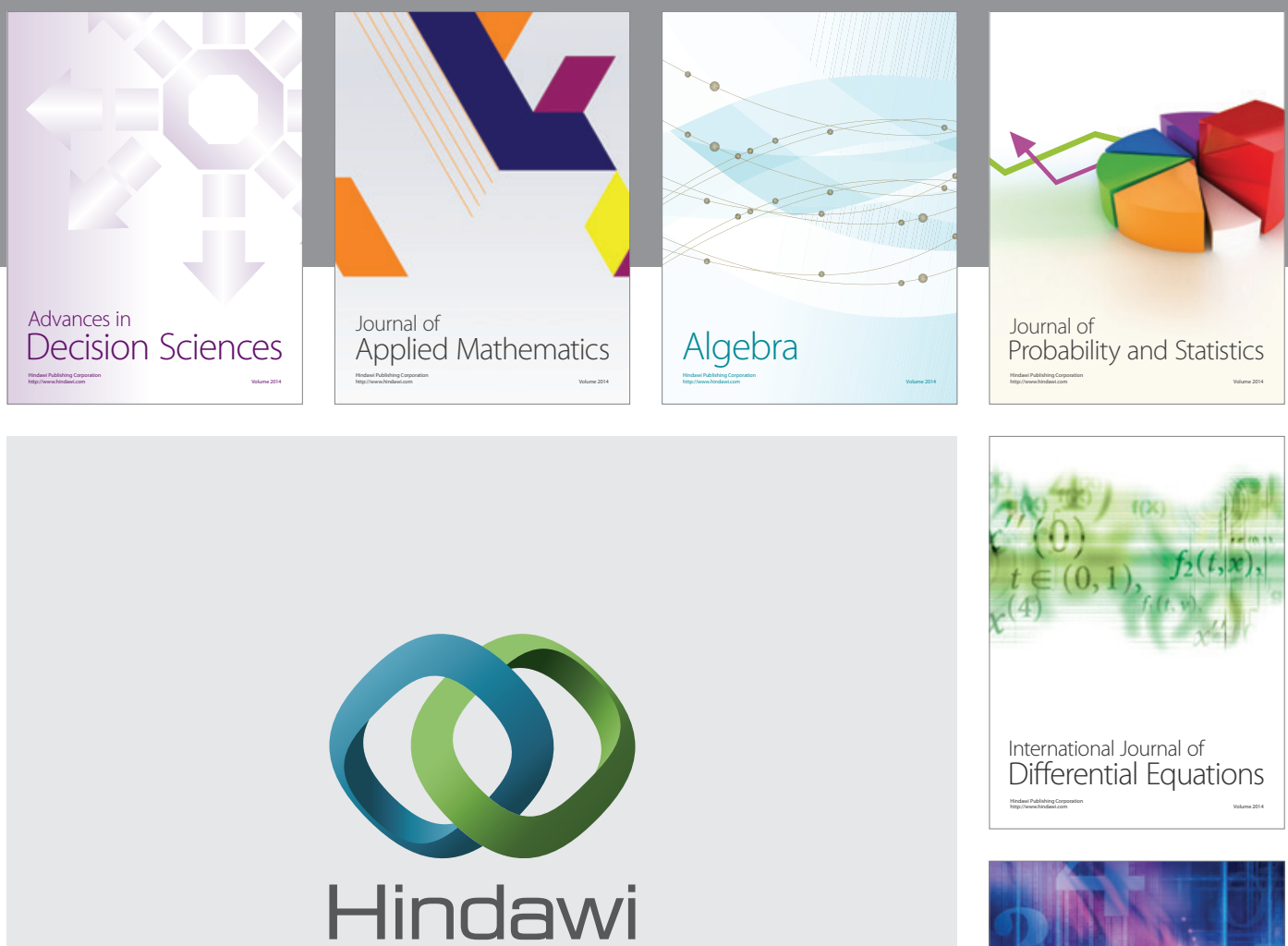

Submit your manuscripts at http://www.hindawi.com
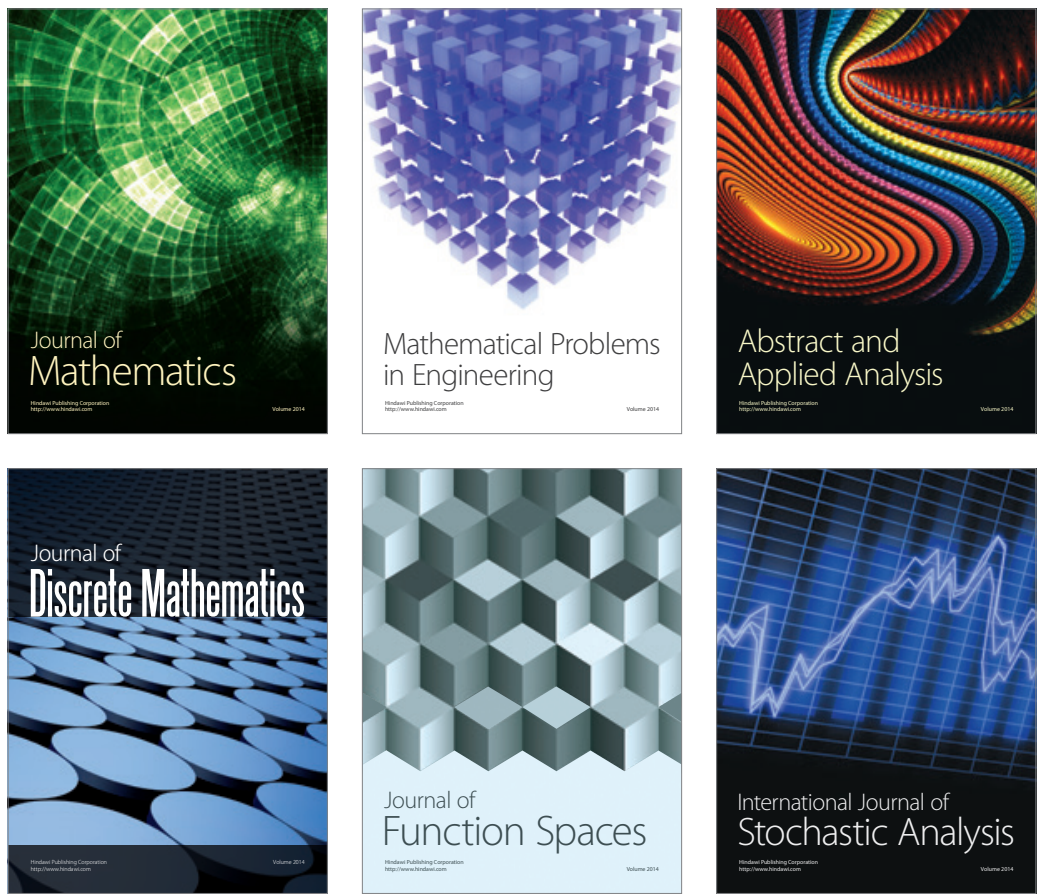

Journal of

Function Spaces

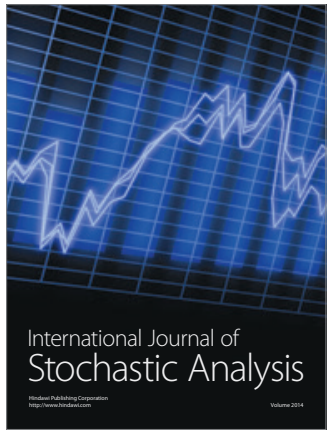

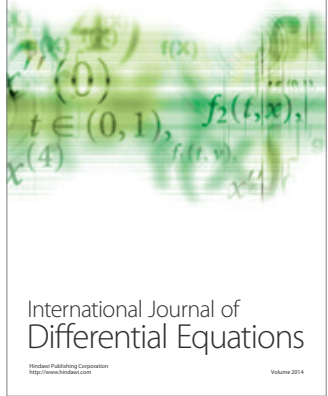
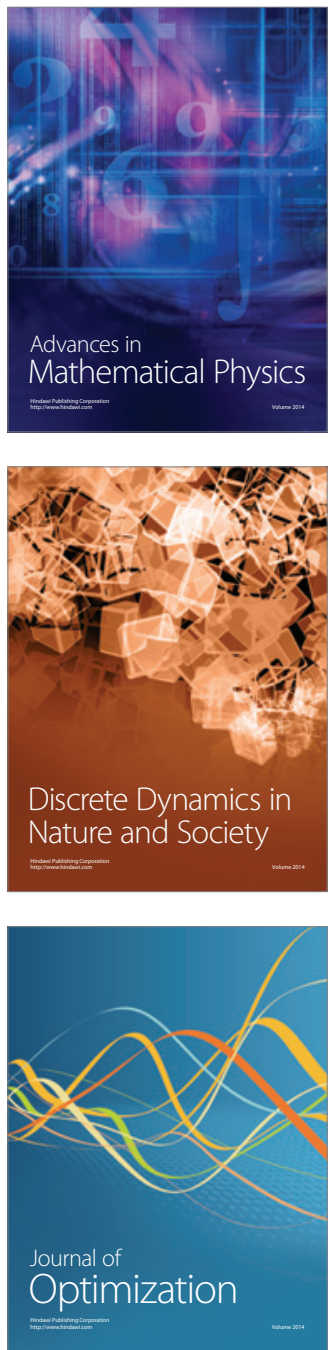